\title{
Geometric interpretation of the effect of the quadrupole force in the collisions of deformed nuclei
}

\author{
M. V. Andrés and J. Gómez-Camacho \\ Departamento de FAMN, Universidad de Sevilla, Facultad de Físicas, Apartado 1065, 41080 Sevilla, Spain \\ M. A. Nagarajan \\ Daresbury Laboratory, Warrington, WA4 4AD, United Kingdom
}

(Received 15 October 1991)

\begin{abstract}
The effect of a quadrupole force on a set of degenerate states of a rotational band with arbitrary spin projection along the symmetry axis $K$ is studied. Analytic expressions for the eigenvalues and eigenvectors are obtained in terms of a set of orthogonal polynomials. This is applied to the collision of a spherical nucleus with a deformed one in which the coupling to a given set of rotational states is allowed, ignoring excitation energies. The elastic $S$-matrix, transition amplitudes, and the fusion cross sections are obtained as a weighted average of the magnitudes corresponding to a set of definite orientations of the axis of the deformed nucleus with respect to the relative coordinate. That weighted average corresponds to approximate the extreme sudden result, consisting of an integral over all the orientations, by a generalized Gaussian quadrature.
\end{abstract}

PACS number(s): 24.10.Eq, 24.70. $+\mathrm{s}, 24.50 .+\mathrm{g}, 03.65 . \mathrm{Nk}$

\section{INTRODUCTION}

The collective excitations of nuclei play a very important role in the reaction mechanisms. The description of these effects requires the explicit inclusion of the excitation by means of coupled-channels calculations. For the case of rotational nuclei, a proper coupled-channels calculation would require the inclusion of many states of the rotational band, making the calculation complicated and time consuming, and complicating the interpretation of the results. On the other hand, when the rotational motion can be considered slow versus the relative motion (sudden approximation) the scattering amplitudes can be calculated as an integral extended to all the orientations of the rotor of the scattering amplitudes calculated as if the orientation of the deformed nucleus was frozen during the reaction, weighted with the probability density that the ground state has that orientation [1]. Also, it is known that when the effect of the coupling to a restricted set of excited states is considered, the excitation energy is ignored, the centrifugal barrier is suitably approximated and the coupling form factors have the same shape, the coupled-channels system can be decoupled, and the scattering amplitudes can be expressed as a combination of the ones corresponding to a set of uncoupled eigenchannels [2-5].

Nagarajan, Balantekin, and Takigawa [6] contributed to bridge the gap between the coupled-channels calculation and the sudden result demonstrating that, for a $K=0$ rotational band, the coupled-channels effect corresponding to include the rotational states from $I=0$ to $I=2 N-2$ ignoring their excitation energies, was equivalent to do a weighted average of the amplitudes corresponding to $N$ orientations. These orientations are characterized by the angle $\theta$ between the symmetry axis of the deformed nucleus and the relative motion, that takes the values such that $P_{2 N}(\cos \theta)=0$. Moreover, that weighted average is precisely the combination obtained when the integral corresponding to the sudden approximation is approximated by an $N$-point Gauss-Legendre quadrature.

The aim of this work is to search for a similar result that could be applied to a rotational band of arbitrary $K$. This is important if one is interested in studying the influence of polarization on the reaction mechanisms, because, if the nucleus is to be polarized, its ground state needs to have spin different from zero.

This paper is organized as follows. In Sec. II we evaluate the matrix element of a quadrupole interaction between rotational states using the tidal spin basis. In Sec. III we map the rotational states into a new set of states characterized by a set of orthogonal polynomials. In Sec. IV we perform an analytical diagonalization in the new basis. In Sec. $V$ we discuss the meaning of eigenvalues and eigenvectors. In Sec. VI we apply this treatment to the calculation of scattering amplitudes. In Sec. VII the relation to the geometrical limit is obtained. Section VIII is for summary and conclusions.

\section{COUPLING POTENTIALS}

Let us consider the interaction between a spherical nucleus and an axially deformed one. The interaction can be written as

$$
V(\mathbf{r}, \hat{\xi})=V_{0}(r)+V_{2}(r) P_{2}(\hat{\mathbf{r}} \cdot \hat{\xi}),
$$

where $\mathbf{r}$ is the relative coordinate and $\hat{\xi}$ stands for the direction of the symmetry axis of the deformed nucleus. In this expression we have neglected spin-orbit terms and hexadecapole and higher-order deformation. The matrix elements of the quadrupole interaction between rotational states in the usual coupled-channels basis is given by 


$$
\begin{aligned}
\left\langle I K L J\left|V_{2}(r) P_{2}(\hat{\mathbf{r}} \cdot \hat{\xi})\right| I^{\prime} K^{\prime} L^{\prime} J\right\rangle & \\
= & V_{2}(r) \delta_{K, K^{\prime}} W\left(I I^{\prime} L L^{\prime} ; 2 J\right) \hat{L} \hat{I} \\
& \times\left\langle L 020 \mid L^{\prime} 0\right\rangle\left\langle I K 20 \mid I^{\prime} K\right\rangle,
\end{aligned}
$$

where $I$ is the internal angular momentum of the deformed nucleus and $K$ is the projection along the symmetry axis, that characterizes the rotational band. $L$ is the orbital angular momentum, and $J$ is the total angular momentum. It is known that this interaction can be partially diagonalized in the tidal spin basis [7]

$$
\begin{aligned}
& \left\langle I K M J\left|V_{2}(r) P_{2}(\hat{\mathbf{r}} \cdot \hat{\xi})\right| I^{\prime} K^{\prime} M^{\prime} J\right\rangle \\
& \quad=V_{2}(r) \delta_{M, M^{\prime}} \delta_{K, K^{\prime}} \hat{I} / \hat{I}^{\prime}\left\langle I M 20 \mid I^{\prime} M\right\rangle\left\langle I K 20 \mid I^{\prime} K^{\prime}\right\rangle,
\end{aligned}
$$

where $M$ is the projection of the spin $I$ along the relative coordinate $\mathbf{r}$ (tidal spin). Note that throughout this paper, $M$ denotes the tidal spin while $K$ is the projection of the spin along the symmetry axis of the rotor. Both magnitudes are conserved by the coupling potential. Note also that the value of $J$ does not affect the values of the coupling potential in the tidal spin basis. So, in what follows, we will drop the index $J$ in the characterization of the states.

The coupling potentials have a common form factor $V_{2}(r)$ and different strength factors. if the excitation energy is ignored for a given number of states, the coupling matrix could be diagonalized numerically, and a set of eigenchannels be obtained, from which all the relevant reaction magnitudes can be calculated [2-5]. However, the eigenvalues and eigenstates of the numerical diagonalization do not have any clear geometrical meaning.

The main difficulty in extending the analytic diagonalization [6] to $K \neq 0$ bands is that each state is coupled to four other states, while for a $K=0$ band it is only coupled to two other. That makes it difficult to make a suitable truncation in the states of the band included in the calculation, and yet obtain analytic expressions for eigenvalues and eigenvectors. An exception to this are the $K=\frac{1}{2}$ bands, because they can be considered as $K=0$ bands to which a $j=\frac{1}{2}$ particle is coupled. For these analytic expressions for eigenvalues and eigenvectors can be found.

\section{MAPPING}

To simplify the complicated coupling structure of a $K \neq 0$ band we will introduce a set of states obtained act-

TABLE I. Mapping coefficients for $K=\frac{3}{2}, M=\frac{3}{2}$.

\begin{tabular}{cccccc}
\hline$\langle n \mid I\rangle$ & $\left|\frac{3}{2}\right\rangle$ & $\left|\frac{5}{2}\right\rangle$ & $\left|\frac{7}{2}\right\rangle$ & $\left|\frac{9}{2}\right\rangle$ & $\left|\frac{11}{2}\right\rangle$ \\
\hline$\langle 0|$ & 1 & & & & \\
$\langle 1|$ & & $\sqrt{\frac{108}{133}}$ & $\sqrt{\frac{25}{133}}$ & & \\
$\langle 2|$ & & $-\sqrt{\frac{110}{2679}}$ & $\sqrt{\frac{792}{4465}}$ & $\sqrt{\frac{342}{517}}$ & $\sqrt{\frac{931}{7755}}$ \\
\hline
\end{tabular}

ing with the $P_{2}(\hat{\mathbf{r}} \cdot \hat{\xi})$ operator over the ground state of the band:

$$
\begin{aligned}
& |0\rangle=|I=K, K, M\rangle \\
& |1\rangle=N_{1}\left(P_{2}|0\rangle-|0\rangle\left\langle 0\left|P_{2}\right| 0\right\rangle\right) \\
& |n\rangle=N_{n}\left(P_{2}|n-1\rangle-\sum_{i=0}^{n-1}|i\rangle\left\langle i\left|P_{2}\right| n-1\right\rangle\right) .
\end{aligned}
$$

These states do not have, in general, good angular momentum $I$. Explicit expressions for overlap $\langle n \mid I K M\rangle$ in the case $K=\frac{3}{2}$ are given in Table $I\left(M=\frac{3}{2}\right)$ and Table II $\left(M=\frac{1}{2}\right)$. The states $|n\rangle$ are the combinations of states $|I K M\rangle$ that are most relevant for the quadrupole coupling. We expect that the coupled-channels effects due to the rotational states $|I K M\rangle$ from $I=K$ to $I=K+2 N$ would be very similar to consider the states $|n\rangle$ from $n=0$ to $n=N$. In both cases, terms of order $N+1$ in $P_{2}$ are ignored: Note that coupling structure is simplified, because each of the states $|n\rangle$ is only coupled to $|n+1\rangle$ and $|n-1\rangle$. This way of generating the $|n\rangle$ states is known as the Lanczos method [8], which allows one to obtain some eigenvalues and eigenstates quite accurately from only part of the full matrix.

Although the states $|n\rangle$ have a complicated expansion in terms of states of good angular momentum, they have a very appealing expression when expressed as a superposition of states corresponding to definite orientations of the rotor. The state $|0\rangle$ can be written as [9]

$$
\begin{aligned}
& |0\rangle=|K K M\rangle \\
& \begin{aligned}
&=\sqrt{(2 K+1) / 16 \pi^{2}} \int d \Omega\left[D_{K M}^{K}(\Omega)|\Omega K\rangle\right. \\
&\left. \pm(-1)^{2 K} \mathcal{D}_{-K M}^{K}(\Omega)|\Omega \bar{K}\rangle\right],
\end{aligned}
\end{aligned}
$$

where $|\Omega K\rangle$ is a state vector of the deformed nucleus corresponding to an orientation given by the Euler angles $\Omega=(\alpha, \beta, \gamma)$ of the rotor with respect to an external coordinate system with the $z$ axis along the relative coordinate, and a projection $K$ of the angular momentum along the symmetry axis. $|\Omega \bar{K}\rangle$ is the time reversed state. We can define the state $|x K M\rangle$ as

$$
\begin{aligned}
|x K M\rangle=N(x) \int d \alpha d \gamma[ & D_{K M}^{K}(\Omega)|\Omega K\rangle \\
& \left. \pm(-1)^{2 K} D_{-K M}^{K}(\Omega)|\Omega \bar{K}\rangle\right],
\end{aligned}
$$

where $x=\cos \beta$ and $N(x)$ is a normalization factor. The states $|x K M\rangle$ have the same parity as the states of the rotational band, and satisfy

TABLE II. Mapping coefficients for $K=\frac{3}{2}, M=\frac{1}{2}$.

\begin{tabular}{lccccc}
\hline$\langle n \mid I\rangle$ & $\left|\frac{3}{2}\right\rangle$ & $\left|\frac{5}{2}\right\rangle$ & $\left|\frac{7}{2}\right\rangle$ & $\left|\frac{9}{2}\right\rangle$ & $\left|\frac{11}{2}\right\rangle$ \\
\hline$\langle 0|$ & 1 & & & & \\
$\langle 1|$ & & $\sqrt{\frac{2}{7}}$ & $\sqrt{\frac{5}{7}}$ & & \\
$\langle 2|$ & & & & $\sqrt{\frac{4}{11}}$ & $\sqrt{\frac{7}{11}}$ \\
\hline \hline
\end{tabular}


TABLE III. Weight functions and orthogonal polynomials.

\begin{tabular}{lcc}
\hline \hline & $K=\frac{3}{2}, M=\frac{3}{2}$ & $K=\frac{3}{2}, M=\frac{1}{2}$ \\
\hline$w(x)$ & $\left(1+3 x^{2}\right) / 4$ & $\left(3-3 x^{2}\right) / 4$ \\
$Q_{0}$ & 1 & 1 \\
$Q_{1}$ & $\sqrt{\frac{7}{152}}\left(15 x^{2}-7\right)$ & $\sqrt{\frac{7}{8}}\left(5 x^{2}-1\right)$ \\
$Q_{2}$ & $\sqrt{\frac{495}{57152}}\left(133 x^{4}-126 x^{2}+17\right)$ & $\sqrt{\frac{55}{64}}\left(21 x^{4}-14 x^{2}+1\right)$ \\
\hline \hline
\end{tabular}

$$
\begin{aligned}
& \mathbf{I} \cdot \hat{\mathbf{r}}|x K M\rangle=M|x K M\rangle, \\
& (\mathbf{I} \cdot \hat{\xi})^{2}|x K M\rangle=K^{2}|x K M\rangle, \\
& P_{2}(\hat{\mathbf{r}} \cdot \hat{\xi})|x K M\rangle=P_{2}(x)|x K M\rangle, \\
& \left\langle x^{\prime} K^{\prime} M^{\prime} \mid x K M\right\rangle=\delta_{K^{\prime}, K^{\prime}} \delta_{M^{\prime}, M} \delta\left(x^{\prime}-x\right) .
\end{aligned}
$$

They can be interpreted as states of the rotor in which the axis of the rotor and the relative coordinate form a fixed angle $\beta$, but they are averaged over all the Euler angles $\alpha$ and $\gamma$ so that $M$ and $K$ are good quantum numbers. In terms of these states, the ground-state wave function is just

$$
|0\rangle=|K K M\rangle=\int_{-1}^{1} d x w(x)^{1 / 2}|x K M\rangle,
$$

where

$$
w(x)=\frac{2 K+1}{4}\left\{\left[d_{K M}^{K}(\beta)\right]^{2}+\left[d_{-K M}^{K}(\beta)\right]^{2}\right\} .
$$

It is straightforward to see that, in general,

$$
|n\rangle=\int_{-1}^{1} d x w(x)^{1 / 2} Q_{n}\left(x^{2}\right)|x K M\rangle,
$$

where $Q_{n}$ is a polynomial of order $n$ that satisfies the orthogonality condition

$$
\langle n \mid m\rangle=\int_{-1}^{1} d x w(x) Q_{n}\left(x^{2}\right) Q_{m}\left(x^{2}\right)=\delta_{n, m} .
$$

Using this equation and the fact that $Q_{0}=1$, one can generate the polynomials. The explicit expressions for $w(x), Q_{0}, Q_{1}$, and $Q_{2}$ are given in Table III, for $K=\frac{3}{2}$ and $M=\frac{1}{2}, \frac{3}{2}$.

Note that, for a $K=0$ band, the state $|n\rangle$ has good angular momentum $I=2 n$. The corresponding orthogonal

polynomials are the even Legendre polynomials. The analytic diagonalization and the geometric interpretation obtained in [6] for the $K=0$ band relied on the orthogonality properties of the Legendre polynomials. Thus, we are in the situation of applying similar techniques for our case.

\section{ANALYTIC DIAGONALIZATION}

We will demonstrate that the (unnormalized) state

$$
|\phi\rangle=\sum_{n=0}^{N} Q_{n}\left(x_{\phi}^{2}\right)|n\rangle
$$

is an eigenstate of the operator $P_{2}(\hat{\mathbf{r}} \cdot \hat{\xi})$ in the subspace generated by $\{|n\rangle, n=0, N\}$, corresponding to the eigenvalue $P_{2}\left(x_{\phi}\right)$, if $x_{\phi}^{2}$ is a zero of $Q_{N+1}$. For that, it should be noticed that the $Q_{n}$, as orthogonal polynomials, satisfy a recursion relation for that can be written as (cf. [10])

$$
\begin{aligned}
x^{2} Q_{n}\left(x^{2}\right)= & a_{n, n+1} Q_{n+1}\left(x^{2}\right)+a_{n, n} Q_{n}\left(x^{2}\right) \\
& +a_{n, n-1} Q_{n-1}\left(x^{2}\right)
\end{aligned}
$$

where $a_{0,-1}=0$. The coefficients in the recursion relation depend on the normalization of the polynomials. In our case, the polynomials are normalized to 1 [see Eq. (14)], it can be seen [10] that $a_{n, n+1}=a_{n+1, n}$ Similarly, one can write

$$
\begin{aligned}
P_{2}(x) Q_{n}\left(x^{2}\right)= & b_{n, n+1} Q_{n+1}\left(x^{2}\right)+b_{n, n} Q_{n}\left(x^{2}\right) \\
& +b_{n, n-1} Q_{n-1}\left(x^{2}\right) .
\end{aligned}
$$

Using that relation, it is straightforward to see that

$$
\sum_{n=0}^{N} Q_{n}\left(y^{2}\right) P_{2}(x) Q_{n}\left(x^{2}\right)=\sum_{n=0}^{N} Q_{n}\left(y^{2}\right) P_{2}(y) Q_{n}\left(x^{2}\right)+b_{N, N+1}\left[Q_{N}\left(y^{2}\right) Q_{N+1}\left(x^{2}\right)-Q_{N}\left(x^{2}\right) Q_{N+1}\left(y^{2}\right)\right]
$$

Using these results, we can write

$$
\begin{aligned}
P_{2}(\hat{\mathbf{r}} \cdot \hat{\xi})|\phi\rangle= & P_{2}\left(x_{\phi}\right)|\phi\rangle \\
& +b_{N, N+1}\left[Q_{N}\left(x_{\phi}^{2}\right)|N+1\rangle\right. \\
& \left.-Q_{N+1}\left(x_{\phi}^{2}\right)|N\rangle\right] .
\end{aligned}
$$

Thus, if the operator is restricted to the subspace generated by $\{|n\rangle, n=0, N\}$ the term proportional to $|N+1\rangle$ cancels. If $x_{\phi}^{2}$ are taken as the zeros of $Q_{N+1}$, the term proportional to $|N\rangle$ vanishes, and we are left with the result we wanted to demonstrate.
Note that $Q_{N+1}$, as an orthogonal polynomial, has $N+1$ zeros in the interval $(0,1)$, which would correspond to $N+1$ eigenvalues and eigenstates of $P_{2}$. The eigenvalues of $P_{2}$ are shown in Tables IV and V. Using the Christoffel-Darboux formula, one gets

$$
\langle\phi \mid \psi\rangle=\sum_{n=0}^{N} Q_{n}\left(x_{\phi}^{2}\right) Q_{n}\left(x_{\psi}^{2}\right)=0
$$

if $x_{\phi}^{2}$ and $x_{\psi}^{2}$ are different zeros of $Q_{N+1}$. Thus, we confirm that the eigenstates are orthogonal. Finally, the eigenstates can be normalized so that 
TABLE IV. Eigenvalues of the $P_{2}$ operator for $K=\frac{3}{2}, M=\frac{3}{2}$.

\begin{tabular}{lrrrrr}
\hline \hline & $\phi=1$ & $\phi=2$ & $\phi=3$ & $\phi=4$ & $\phi=5$ \\
\hline$N=0$ & 0.2000 & & & & \\
$N=1$ & -0.2556 & 0.6766 & & & \\
$N=2$ & -0.3871 & 0.2303 & 0.8294 & & \\
$N=3$ & -0.4367 & -0.0312 & 0.4984 & 0.8953 & \\
$N=4$ & -0.4599 & -0.1811 & 0.2385 & 0.6512 & 0.9294 \\
\hline \hline
\end{tabular}

$$
\begin{aligned}
& |\phi\rangle=\sum_{n=0}^{N}|n\rangle\langle n \mid \phi\rangle, \\
& \langle n \mid \phi\rangle=Q_{n}\left(x_{\phi}^{2}\right)\left(\sum_{i=0}^{N} Q_{i}^{2}\left(x_{\phi}^{2}\right)\right)^{-1 / 2} .
\end{aligned}
$$

\section{INTERPRETATION OF THE EIGENVALUES AND EIGENSTATES}

It should be noticed that the states $|n\rangle$ form a complete basis of all the combinations of states $|x K M\rangle$ that are even in $x$. Thus, a state $\sqrt{\frac{1}{2}}(|x K M\rangle+|-x K M\rangle)$, that correspond to an axially symmetric deformed nucleus whose symmetry axis forms a fixed angle with the relative coordinate, can be expanded in terms of the states $|n\rangle$ as

$$
\sqrt{\frac{1}{2}}(|x K M\rangle+|-x K M\rangle)=\sqrt{2 w(x)} \sum_{n=0}^{\infty} Q_{n}\left(x^{2}\right)|n\rangle .
$$

If this expansion is truncated up to $n=N$, we will have the state in the subspace generated by the basis $\{|n\rangle, n=0, N\}$ that resembles most closely to a state with a fixed orientation. This truncation is more accurate for the values of $x$ so that $Q_{N+1}\left(x^{2}\right)=0$. Thus, we can interpret the eigenstates of $P_{2}(\hat{\mathbf{r}} \cdot \hat{\xi})$ in a subspace generated by $\{|0\rangle \cdots|N\rangle\}$ as the combination of states that resemble most to states of a definite orientation. This interpretation is strengthened by the fact that the eigenvalues of $P_{2}(\hat{\mathbf{r}} \cdot \hat{\xi})$ in the truncated subspace coincide with those corresponding to the states of definite orientation $\left|x_{\phi} K M\right\rangle$, that are $P_{2}\left(x_{\phi}\right)$.

To illustrate this, we can express the states $|n\rangle$ as a combination of states corresponding to an angle $\beta$ between the axis of the rotor and the relative coordinate:

$$
|n\rangle=\int_{0}^{\pi} d \beta f_{n}(\beta)|\beta K M\rangle
$$

The values of $\left|f_{n}(\beta)\right|^{2}$ represent the probability density of having an angle $\beta$ between the axis of the rotor and the relative coordinate. They are plotted in Figs. 1 and 2 for $K=\frac{3}{2}, n=0,1,2$. They do not present any preferred orientation, and resemble the qualitative behavior of the modulus square of spherical harmonics.

If we use the equivalent expression for the eigenstates $|\phi\rangle$,

$$
|\phi\rangle=\int_{0}^{\pi} d \beta f_{\phi}(\beta)|\beta K M\rangle
$$

we find that the values of $\left|f_{\phi}(\beta)\right|^{2}$ (see Figs. 3 and 4) have significant values at orientations close to the angles $\beta_{\phi}$ such that their cosines give the zeros of $Q_{N+1}\left(x^{2}\right)$. As $N$ gets bigger, the function $\left|f_{\phi}(\beta)\right|^{2}$ becomes a $\delta$ function.

\section{APPLICATION TO SCATTERING IN THE SUDDEN LIMIT}

The interaction between the nuclei conserves both the tidal spin $M$ and the projection of the spin along the symmetry axis of the rotor $K$. However, that is not true for the full Hamiltonian. The centrifugal term of the relative motion changes $M$, while the Coriolis term of the internal Hamiltonian of the rotor changes $K$. However, the Coriolis term can be neglected for not very high internal angular momentum $I$, and the centrifugal term can be substituted by an average value (isocentrifugal approximation [7]) for heavy-ion collisions. Note that, when the ground-state angular momentum $I=0$ and one is discussing elastic scattering, the isocentrifugal approximation is equivalent to ignoring the Coriolis force of the relative motion [2]. The isocentrifugal approximation implies that $M$ is conserved in the scattering process, while neglecting the Coriolis force of the internal degrees of freedom implies that $K$ is conserved.

Any magnitude related to the scattering of two particles can be obtained in terms of the $S$ matrix. This, in

TABLE V. Eigenvalues of the $P_{2}$ operator for $K=\frac{3}{2}, M=\frac{1}{2}$.

\begin{tabular}{lccccc}
\hline & $\phi=1$ & $\phi=2$ & $\phi=3$ & $\phi=4$ & $\phi=5$ \\
\hline$N=0$ & -0.2000 & & & & \\
$N=1$ & -0.3780 & 0.3780 & & & \\
$N=2$ & -0.4343 & 0.0252 & 0.6399 & & \\
$N=3$ & -0.4590 & -0.1574 & 0.3187 & 0.7683 & \\
$N=4$ & -0.4720 & -0.2606 & 0.1008 & 0.5068 & 0.8393 \\
\hline \hline
\end{tabular}




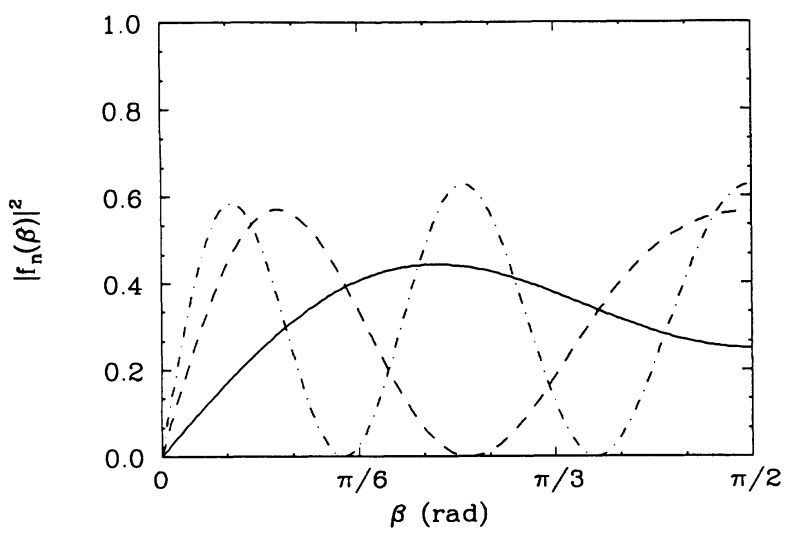

FIG. 1. $\left|f_{n}(\beta)\right|^{2}$ vs the angle $\beta$ for $K=\frac{3}{2}, M=\frac{1}{2}$. The solid line corresponds to $n=0$, the dashed line to $n=1$, and the dotdashed line to $n=2$.

general, will be a function of the total angular momentum $J$, the incoming and outgoing orbital angular momenta $L, L^{\prime}$ the incoming and outgoing intrinsic angular momenta $I, I^{\prime}$, and their projection along the symmetry axis $K, K^{\prime}$. When the Coriolis term can be ignored, the $S$ matrix is diagonal in $K$. Besides, when tidal forces dominate, and the isocentrifugal approximation can be done [7], the $S$ matrix elements can be written in terms of the tidal spin $S$ matrices as

$S_{L^{\prime} I^{\prime} K^{\prime}, L I K}^{J}$

$$
=\delta_{K, K^{\prime}} \mathrm{Ph} \frac{\hat{L} \hat{L}^{\prime}}{\hat{J}^{2}} \sum_{M}\langle L 0 I M \mid J M\rangle\left\langle L^{\prime} 0 I^{\prime} M \mid J M\right\rangle S_{I^{\prime}, I}^{\bar{L} M K},
$$

where $M$ is the tidal spin, $\bar{L}=\left(L+L^{\prime}\right) / 2$, and $\mathrm{Ph}$ is a phase factor involving Coulomb phase shifts. Now, if we consider the coupling of the rotational states from $I=K$ to $K+2 N$, ignoring the excitation energies, we can use the mapping discussed previously to get

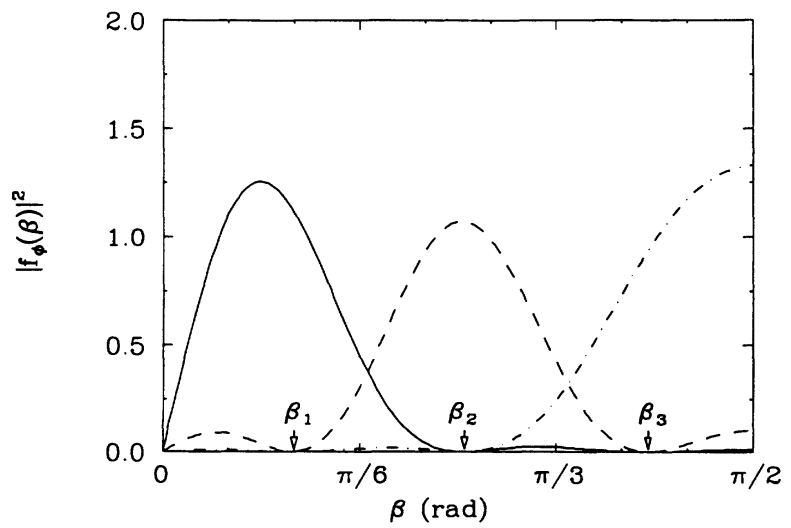

FIG. 3. $\left|f_{\phi}(\beta)\right|^{2}$ vs the angle $\beta$ for $K=\frac{3}{2}, M=\frac{1}{2}$. The solid line corresponds to $\phi=0$, the dashed line to $\phi=1$, and the dotdashed line to $\phi=2$.

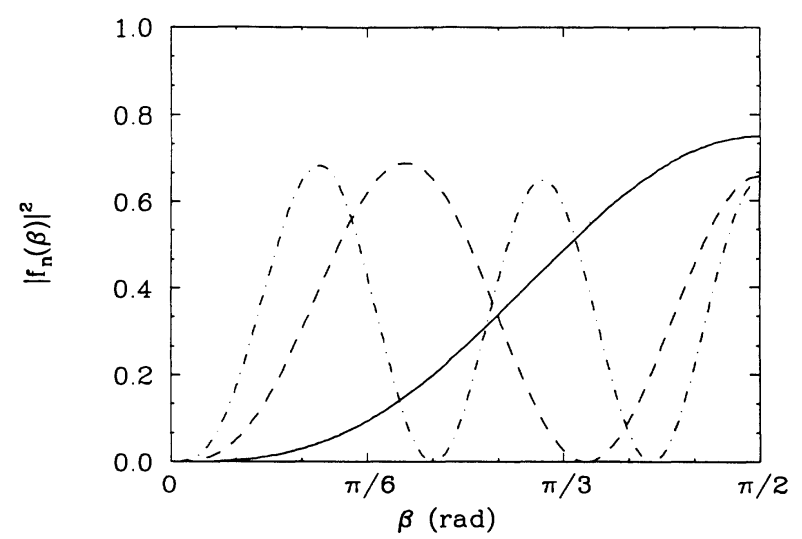

FIG. 2. $\left|f_{n}(\beta)\right|^{2}$ vs the angle $\beta$ for $K=\frac{3}{2}, M=\frac{3}{2}$. Same notation as Fig. 1.

$$
S_{I^{\prime}, I}^{\bar{L} M K}=\sum_{n, n^{\prime}=0}^{N}\left\langle I^{\prime} K M \mid n^{\prime}\right\rangle S_{n^{\prime}, n}^{\bar{L} M K}\langle n \mid I K M\rangle .
$$

The coefficients $\langle n \mid I K M\rangle$ can be obtained in a straightforward (but laborious) way from the definition of the states $|n\rangle$. Some of them are presented in Tables I and II.

Now if one uses the basis $|\phi\rangle$ that diagonalizes the interaction, it will also diagonalize the $S$ matrix, and one gets

$$
S_{n^{\prime}, n}^{\bar{L} M K}=\sum_{\phi=1}^{N+1}\left\langle n^{\prime} \mid \phi\right\rangle S^{\bar{L}}\left(x_{\phi}\right)\langle\phi \mid n\rangle .
$$

The $S$ matrix $S^{\bar{L}}\left(x_{\phi}\right)$ is the one obtained from a onechannel optical model calculation with the potential

$$
V_{\phi}(r)=V_{0}(r)+V_{2}(r) P_{2}\left(x_{\phi}\right) .
$$

The elastic $S$ matrix for the ground state is just

$$
S_{K, K}^{\bar{L} M K}=\sum_{\phi=1}^{N+1} w_{\phi}^{M K} S^{\bar{L}}\left(x_{\phi}\right),
$$

where

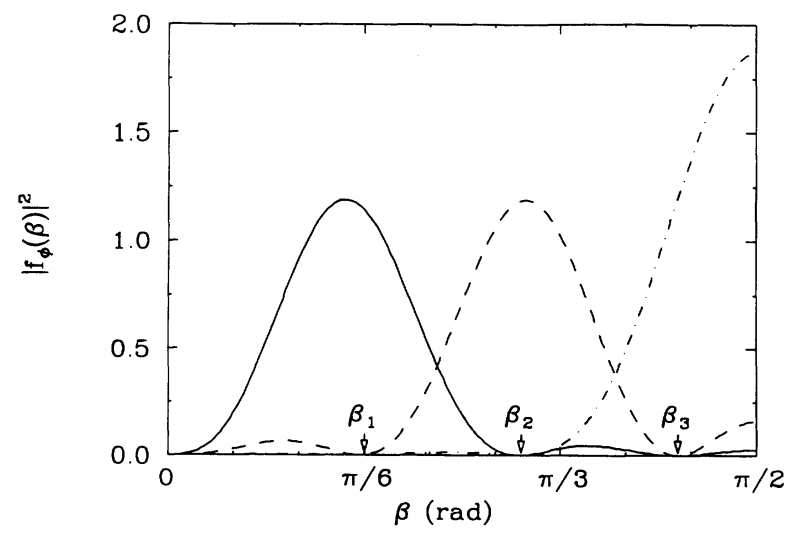

FIG. 4. $\left|f_{\phi}(\beta)\right|^{2}$ vs the angle $\beta$ for $K=\frac{3}{2}, M=\frac{3}{2}$. Same notation as Fig. 3. 
TABLE VI. Weight factors for $K=\frac{3}{2}, M=\frac{3}{2}$.

\begin{tabular}{lccccc}
\hline \hline & $\phi=1$ & $\phi=2$ & $\phi=3$ & $\phi=4$ & $\phi=5$ \\
\hline$N=0$ & 1.0000 & & & & \\
$N=1$ & 0.5113 & 0.4887 & & & \\
$N=2$ & 0.3145 & 0.4120 & 0.2735 & & \\
$N=3$ & 0.2238 & 0.2973 & 0.3068 & 0.1721 & \\
$N=4$ & 0.1733 & 0.2218 & 0.2587 & 0.2286 & 0.1176 \\
\hline \hline
\end{tabular}

$$
w_{\phi}^{M K}=\left(\sum_{i=0}^{N} Q_{i}^{2}\left(x_{\phi}^{2}\right)\right]^{-1}
$$

Note that $\Sigma_{\phi} w_{\phi}^{M K}=1$. The weight factors $w_{\phi}^{M K}$ are shown in Tables VI and VII. As the weight factors do not depend upon $\bar{L}$, the sum to obtain the scattering amplitudes as a function of the scattering angle for a given tidal spin can be performed, and one gets

$$
A_{K, K}^{M}(\theta)=\sum_{\phi=1}^{N+1} w_{\phi}^{M K} A\left(x_{\phi}, \theta\right)
$$

Note that tidal symmetry leads to the fact that the transition amplitudes corresponding to projections $m, m^{\prime}$ of the spin along the beam direction are given by

$$
A_{K m, K m^{\prime}}(\theta)=\sum_{M} d_{m, M}^{K}\left(\frac{\pi+\theta}{2}\right) A_{K, K}^{M}(\theta) d_{m^{\prime}, M}^{K}\left(\frac{\pi+\theta}{2}\right) \text {. }
$$

The fusion cross sections, defined as the cross section that does not appear in the channels included explicitly in the calculation, for a given value the angular momentum $J$ and of the tidal spin $M$ can be calculated in terms of those of the uncoupled eigenchannels and one gets

$$
\sigma_{K}^{J M}=\sum_{\phi=1}^{N+1} w_{\phi}^{M K} \sigma^{J}\left(x_{\phi}\right) .
$$

Note that the fusion cross sections for a given projection of the spin of the projectile along the beam direction $m$ can be related to the fusion cross sections for a given tidal spin $M$ using tidal symmetry [11]

$$
\sigma_{K, m}^{J}=\sum_{M}\left|d_{m, M}^{K}\left(\frac{\pi+\theta_{J}}{2}\right)\right|^{2} \sigma_{K}^{J M},
$$

where $\theta_{J}$ is the classical scattering angle that corresponds to an angular momentum $J$.

\section{RELATION TO THE GEOMETRICAL LIMIT}

If the excitation energy of all the states of the rotational band can be ignored, which is equivalent to assuming that the moment of inertia of the rotor is very large, and so the orientation of the rotor is fixed during the scattering process, the scattering amplitudes will be given in terms of the average over all the orientations, weighted with the probability density that they occur in the ground state [1]:

$$
\begin{aligned}
& S_{K, K}^{\bar{L} M}=\int_{-1}^{1} d x w(x) S^{\bar{L}_{(}}(x), \\
& A_{K, K}^{M}(\theta)=\int_{-1}^{1} d x w(x) A(x, \theta), \\
& \sigma_{K}^{J M}=\int_{-1}^{1} d x w(x) \sigma^{J}(x) .
\end{aligned}
$$

Let $P_{m}(x)$ be a set of polynomials that are orthogonal with respect to the weight function $w(x)$. One can approximate the integrals as follows:

$$
\int_{-1}^{1} d x w(x) f(x) \approx \sum_{\phi=1}^{N^{\prime}+1} A_{\phi} f\left(x_{\phi}\right),
$$

where $x_{\phi}$ are the zeros of the polinomial $P_{M+1}(x)$, and $A_{\phi}$ are weights given by the expression

$$
A_{\phi}=\int_{-1}^{1} d x w(x) L_{\phi}(x) \text {. }
$$

$L_{\phi}(x)$ is the Lagrange multiplier function [12] that can be written as

$$
L_{\phi}(x)=\frac{\sum_{m=0}^{N^{\prime}} P_{m}\left(x_{\phi}\right) P_{m}(x)}{\sum_{m=0}^{N^{\prime}} P_{m}\left(x_{\phi}\right)^{2}} .
$$

Using the orthogonality of $P_{m}(x)$ one gets

$$
A_{\phi}=\left(\sum_{m=0}^{N^{\prime}} P_{m}\left(x_{\phi}\right)^{2}\right)^{-1}
$$

The truncation error is of the order of the $2 N^{\prime}+2$ derivative of $f(x)$.

In the case that $w(x)$ and $f(x)$ are even functions of $x$, only the polynomials $Q_{n}\left(x^{2}\right)$ are relevant, and one can write the expression

TABLE VII. Weight factors for $K=\frac{3}{2}, M=\frac{1}{2}$.

\begin{tabular}{lccccc}
\hline \hline & $\phi=1$ & $\phi=2$ & $\phi=3$ & $\phi=4$ & $\phi=5$ \\
\hline$N=0$ & 1.0000 & & & & \\
$N=1$ & 0.7646 & 0.2354 & & & \\
$N=2$ & 0.5916 & 0.3325 & 0.0759 & & \\
$N=3$ & 0.4779 & 0.3380 & 0.1532 & 0.0309 & \\
$N=4$ & 0.3995 & 0.3167 & 0.1911 & 0.0779 & 0.0147 \\
\hline \hline
\end{tabular}




$$
\int_{-1}^{1} d x w(x) f(x) \approx \sum_{\phi=1}^{N+1} B_{\phi} f\left(x_{\phi}\right),
$$

where $x_{\phi}$ are the positive zeros of $Q_{N+1}\left(x^{2}\right)$, and

$$
B_{\phi}=\int_{-1}^{1} d x w(x) L_{\phi}\left(x^{2}\right)=\left[\sum_{n=0}^{N} Q_{n}\left(x_{\phi}^{2}\right)^{2}\right]^{-1} .
$$

The truncation error is of the order of the $4 N+4$ derivative of $f(x)$.

Comparing these expressions with the ones obtained before, we conclude that the effect of including the coupling of the states $|n\rangle$ from $n=0$ to $N$, or the rotational states $|I K M\rangle$ from $I=K$ to $K+2 N$, ignoring the excitation energies, is equivalent to performing an $(N+1)$ point Gaussian integration on the geometrical expressions of these magnitudes. Moreover, the coupling to the states $I=K+2 N+1$ and $I=K+2 N+2$ is going to be important for a magnitude such as the elastic scattering or the reaction cross section when the geometrical expression of that magnitude as a function of the orientation angle has a significant contribution of the $4 N+4$ derivative.

\section{SUMMARY AND CONCLUSIONS}

The quadrupole coupling in a rotational band with $K \neq 0$ can be simplified if the states with definite angular momentum are mapped into a set of states $|n\rangle$ obtained acting with the $P_{2}$ operator on the rotational ground state and orthogonalizing. These states correspond to a superposition of states with definite orientations, weighted with a set of orthogonal polynomials. In general, the states $|n\rangle$ do not correspond to states of good angular momentum and hence the truncation of the basis states $|n\rangle$ is, except for the case of $K=0$ and $K=\frac{1}{2}$ bands, distinct from the truncation in terms of the members of the ground-state rotational band. Nevertheless, the results obtained when truncating in these two different ways are very similar, as it can be seen in the Appendix.

The eigenvalues and eigenvectors of the $P_{2}$ operator in basis defined by the states $|n\rangle$ from $n=0$ to $N$ are obtained in terms of the zeros of the orthogonal polynomial of order $N+1$. The eigenvectors can be interpreted as the combination of states $|n\rangle$ from $n=0$ to $N$ that most resemble a state with definite orientation of the symmetry axis of the rotor with respect to the relative coordinate. The eigenvalues correspond to $P_{2}(x)$, where $x$ is the cosine of the orientation angle.

The $S$ matrix, elastic transition amplitudes, and fusion cross sections in the tidal spin basis correspond to a weighted average of these magnitudes corresponding to the orientations that define the eigenvalues and eigenvectors of $P_{2}$.

The classical sudden result for these magnitudes correspond to an integral over all the orientation angles, weighted with the probability that a given orientation occurs in the ground state. The weighted average described before corresponds to a generalized Gaussian quadrature of the sudden integral expression.

The effect of the inclusion of the $2 N+1$ and $2 N+2$ states of the rotational band in a coupled-channels calculation will affect significantly the elastic magnitudes ( $S$ matrix and transition amplitudes) and the fusion cross sections when the parametric expression of these magnitudes as a function of the cosine of the orientation angle presents significant contributions of the $4 N+4$ derivative.

As a final comment, we think that the analytic diagonalization found in this work and in other cases discussed in [6] is not just a mathematical curiosity. One should expect to have it for other kinds of collective excitation. An algebraic treatment of the diagonalization of the collective excitation, based on group theory, can be an alternative to the analytic approach presented here, based on the properties of the orthogonal polynomials, and it can provide a deeper understanding of the effect of internal degrees of freedom on the reaction mechanisms.

This work was partially supported by the Acciones Integradas HB-196 and the Spanish DGICYT PB89-0636.

\section{APPENDIX: COMPARISON OF THE ANALYTIC RESULTS WITH NUMERICAL DIAGONALIZATION}

The coupling matrix of Eq. (3) can be diagonalized numerically, including the rotational states $|I K M\rangle$ from $I=K$ to $I=K+2 N$. Thus, one obtains $2 N+1$ eigenvalues and eigenvectors, from which the scattering magnitudes can be obtained. However, the analytical diagonalization including the states $|n\rangle$ from $n=0$ to $N$ generates $N+1$ eigenvalues and eigenvectors. Despite this fact, we will show that the predictions for scattering magnitudes happen to very similar in both cases.

When $K$ or $M$ take the value 0 , only the values of $I$ with $I-K$ even are coupled. The states $|n\rangle$ coincide with the states of a given angular momentum $I=K+2 n$. When $K$ or $M$ take the value $\frac{1}{2}$, of the $2 N+1$ eigenstates of $P_{2}, N$ are orthogonal to the ground state, and hence do not affect the reaction mechanism. The remaining $N+1$ coincide with the eigenstates in the basis $|n\rangle$ and have the same eigenvalues. This is due to the fact that rotational states with $K$ (or $M$ ) equal to $\frac{1}{2}$ can be considered as rotational states with $K$ or $M$ equal to 0 to which a particle with $j=\frac{1}{2}$ is coupled. For the other cases, the eigenstates and eigenvalues in the $|I K M\rangle$ basis differ from those in the $|n\rangle$ basis.

Let us consider the Wong formula for the fusion cross section

$$
\sigma_{f}=\frac{\hbar \omega R_{b}^{2}}{2 E} \ln \left[1+\exp \left[2 \pi \frac{E-V_{b}}{\hbar \omega}\right)\right] .
$$

The barrier height depends on the orientation of the rotor with respect to the relative coordinate as $V_{b}=V_{0}+V_{2} P_{2}(\cos \theta)$. Hence, within the sudden approximation, the fusion cross section will be given by

$$
\begin{aligned}
\sigma_{f}=\sum_{i=1}^{N_{c}} & w_{i} \frac{\hbar \omega R_{b}^{2}}{2 E} \\
& \times \ln \left[1+\exp \left[2 \pi \frac{E-V_{0}-V_{2} P_{2}^{i}}{\hbar \omega}\right]\right],
\end{aligned}
$$


TABLE VIII. Enhancement factors for the fusion cross section calculated in the $|n\rangle$ basis and in the $|I K M\rangle$ basis.

\begin{tabular}{lccccc}
\hline \hline & \multicolumn{3}{c}{$E \ll V_{0}$} & \multicolumn{2}{c}{$E=V_{0}$} \\
$N$ & $I_{\max }$ & $F(n)$ & $F(I)$ & $F(n)$ & $F(I)$ \\
\hline 0 & $\frac{3}{2}$ & 7.3891 & 7.3891 & 3.0685 & 3.0685 \\
1 & $\frac{7}{2}$ & 424.29 & 488.36 & 4.8265 & 4.7463 \\
2 & $\frac{11}{2}$ & 1098.3 & 1150.6 & 4.7079 & 4.7081 \\
3 & $\frac{15}{2}$ & 1375.9 & 1390.3 & 4.6719 & 4.6784 \\
4 & $\frac{19}{2}$ & 1435.2 & 1437.4 & 4.6989 & 4.6950 \\
\hline \hline
\end{tabular}

where $P_{2}^{i}$ is the $i$ th eigenvalue of $P_{2}$ and $w_{i}$ is the square of the overlap of the ground state with the $i$ th eigenstate. Let us define an enhancement factor $F$ as the ratio of the coupled-channels fusion cross section to the uncoupled cross sections. When the energy is well below the barrier, that factor is given by

$$
F=\sum_{i=1}^{N_{c}} w_{i} \exp \left[-\frac{2 \pi V_{2}}{\hbar \omega} P_{2}^{i}\right] \text {. }
$$

When the energy coincides with the underformed barrier $V_{0}$

[1] D. M. Chase, Phys. Rev. 104, 838 (1956).

[2] P. Jacobs and U. Smilansky, Phys. Lett. 127B, 313 (1983).

[3] C. H. Dasso, S. Landowne, and A. Winther, Nucl. Phys. A405, 381 (1983); A407, 221 (1983); A432, 555 (1985).

[4] R. Lindsay and N. Rowley, J. Phys. G 10, 805 (1984).

[5] M. A. Nagarajan, in Proceedings of the Second La Rábida Summer School on Nuclear Structure and Nuclear Reactions, edited by M. Lozano and G. Madurga (World Scientific, Singapore, 1986), p. 349.

[6] M. A. Nagarajan, B. Balantekin, and N. Takigawa, Phys. Rev. C 34, 894 (1986).

$$
F=\sum_{i=1}^{N_{c}} w_{i} \ln \left[1+\exp \left(-\frac{2 \pi V_{2}}{\hbar \omega} P_{2}^{i}\right)\right] / \ln 2
$$

In Table VIII we present the evaluation of the factor $F$ for a nucleus with $K=M=\frac{3}{2}$, calculated in the basis $|n\rangle$ and in the basis $|I K M\rangle$. We have taken $2 \pi V_{2} / \hbar w=10$, that is reasonable for the system ${ }^{23} \mathrm{Na}+{ }^{208} \mathrm{~Pb}$. The agreement is remarkable, and justifies the mapping performed in Sec. III.

[7] J. Gómez-Camacho and R. C. Johnson, J. Phys. G 12, L235 (1986); 14, 609 (1988).

[8] P. J. Brussard and P. W. M. Glaudemans, Shell-Model Applications in Nuclear Spectroscopy (North-Holland, Amsterdam, 1977), p. 371.

[9] D. M. Brink et al., J. Phys. G 13, 629 (1987).

[10] M. Abramowitz and I. A. Stegun, Handbook of Mathematical Functions (Dover, New York, 1965), p. 773.

[11] J. Gómez-Camacho, Phys. Lett. B 185, 310 (1987).

[12] F. Scheid, Theory and Problems of Numerical Analysis (McGraw-Hill, New York, 1968), p. 125. 